\title{
Explainable Artificial Intelligence and its potential within Industry
}

\author{
Saad Mahamood \\ saad.mahamoodetrivago.com
}

trivago N.V., Düsseldorf, Germany

\begin{abstract}
The age of Big Data has enabled the creation of artificial intelligence solutions that has allowed systems to better respond to their users requests and needs. Applications such as recommender systems, automated content generation systems, etc. are increasingly leveraging such large amounts of data to make better informed decisions about how to tailor their output appropriately. However, the opaqueness of these AI systems in how they derive their decisions or outputs has led to an increasing call for transparency with increasing concerns for the potential of bias to occur in areas such as finance and criminal law. The culmination of these calls have lead to tentative legislative steps. For example, the "Right to explanation" as part of the recently enacted European Union's General Data Protection Regulation.

Natural Language Generation (NLG) has been used in successfully in many data-to-text applications allowing users to gain insights from their data sets. Whilst NLG technology has a strong role to play in generating explanations for AI models there still remains inherit challenges in developing and deploying text generation systems within a commercial context.

In this talk I will explore the role and potential that Natural Language Explainable AI can have within trivago and the wider industry. trivago is a leading accommodation meta-search engine that enables users find the right hotel or apartment at the right price. In particular, this talk will describe the work we have done to apply natural language solutions within trivago and the challenges of applying AI solutions from a commercial perspective. Finally, this talk will also explore the potential applications of where explainable AI approaches could be used within trivago.
\end{abstract}


